\title{
Temporally resolved analysis of the response of density and temperature to modulated central electron heating on ASDEX Upgrade
}

\author{
A. Mlynek, C. Angioni, E. Fable, R. Fischer, F. Ryter, J. Stober, W. Suttrop, H. Zohm and the ASDEX Upgrade Team \\ Max-Planck-Institut für Plasmaphysik, 85748 Garching, Germany
}

\begin{abstract}
In the recent years, it has been found that the application of local heat sources in a fusion plasma can influence the shape of the density profile. This shows that a coupling between heat and particle transport exists, which is taken into account in transport models by introducing off-diagonal transport coefficients. In existing studies, the focus of analysis is mostly on the density profile that occurs after a sufficiently long settling time after altering the heating power level. In this article, the transient behavior of temperature and density in response to steps in the applied heating power is analyzed, which leads to the observation of hysteresis loops in the local density-temperature diagram. Based on numerical transport simulations, it is shown that the occurrence of hysteresis curves can be reproduced on the basis of today's physics understanding, where the shape of the curve provides additional information on the transport coefficients and their interdependences.
\end{abstract}

\section{INTRODUCTION}

The spatial distribution of density in a fusion experiment is of significant importance as it enters in numerous analyses and substantially contributes to the fusion performance. Despite of the fact that particle fueling is mostly peripheral, centrally peaked density profiles are regularly observed in tokamak discharges. Density peaking in the hot plasma center is beneficial for achieving high fusion rates. A profound understanding of the underlying transport phenomena is therefore mandatory in order to reliably predict the peakedness of the density profile in next-step fusion devices, and finally their achievable fusion power.

It has been shown in many experiments that the application of local heat sources in the plasma can influence the shape of the density profile under certain conditions [1] [2] [3]. In low-density L-mode discharges, for example, the application of electron heating at or close to the magnetic axis causes a reduction of the core density and thus a flattening of the density profile. This effect is often referred to as density 'pump-out' in the literature. Depending on the global plasma parameters, the effect can also reverse sign, resulting in an increase of the density peaking in response to central heating. It was recently found that the effective collisionality is a key parameter for characterizing the density profile response to local heating [4] [5].

In existing studies on the impact of heating on the density profile, usually the static density profile is analyzed which occurs after sufficiently long settling time after switching the heating power to a new level. In this article, however, the focus will be on the transient behavior of temperature and density in phases where the heating power is increased or decreased in steps. In the 2009 ASDEX Upgrade experimental campaign, a new diagnostic came into operation which yields a density profile in real-time that is calculated from the line-integrated measurements of 5 horizontal interferometer channels by a fast deconvolution algorithm [6]. The new capabilities provided by this diagnostic were used to feedback control the shape of the density profile in real-time, which resulted in the need for an actuator that is able to modify the spatial distribution of density. It was decided to use central electron cyclotron resonance heating $(\mathrm{ECRH})$ for this purpose, making use of the density pump-out effect. In preparatory experiments, series of identical ECRH pulses were injected into the plasma in order to test the reproducibility of the effect and to explore the achievable amount of flattening. The data collected in these discharges contains a large number of transient phases for both, rising and falling edges in the applied ECRH power, and therefore turned out to be an excellent basis for temporally resolved heat and particle transport studies. These studies show that at a given radial location in the plasma, the curve along which the system evolves in the local density-temperature diagram is not a straight line, but a hysteresis loop when ECRH is switched on and off periodically.

It will be shown that this transient behavior can be reproduced with the ASTRA simulation code on the basis of the transport equations for heat and particles. The occurrence of a hysteresis curve is predicted by the simulation for practically any setting that can be made for the transport coefficients, however, its shape clearly depends on the choice of those coefficients. The analysis of the transient behavior observed in the experiments can therefore yield additional constraints on the transport coefficients and their interdependences which one could not obtain from the pure analysis of the stationary profiles of density and temperature occurring after some settling time.

The second section of this article describes the experimental studies that have been carried out on ASDEX Upgrade and deals with factors which might bias the observed results and therefore have to be avoided. The third section is dedicated to the analysis of the transient behavior of density and temperature in the experiments. Section four presents ASTRA simulations which model the temporal evolution of temperature and density profile on the basis of the transport equations for heat and particles, and section five summarizes the results and gives 
an outlook to future studies.

\section{EXPERIMENTS ON ASDEX UPGRADE}

As detailed in the previous section, the experiments on which this study is based were primarily carried out with the aim of exploring the capabilities of ECRH as an actuator that allows for modification of the shape of the density profile. Aspects of particular interest were the achievable amount of flattening or peaking of the density profile, the speed at which the profile changes and the reproducibility of the process. With respect to the last point, there was the concern that after a heating cycle, which consists of an ECRH pulse and a subsequent phase of equal length without ECRH, the plasma might not fully return to the state before the heating pulse. That way, the plasma parameters might continuously drift in one direction during a sequence of heating pulses, which can in turn change the density response to the heating pulses. For this reason, always a series of identical heating pulses was injected into the plasma. If the time traces of density and temperature are strictly periodic in this phase, i.e. return to the same level at the end of each heating cycle, one can conclude that no such drift is present. This series of heating pulses used in the experiments turns out to be an excellent basis for the transient studies presented in this article.

Experiments in both, L-mode and H-mode discharges have been performed. In literature that is based on earlier ASDEX Upgrade experiments, a strong density pump-out effect is reported for low-density L-mode discharges [3]. Accordingly, L-mode discharges at lineaveraged densities of $2-3 \cdot 10^{19} m^{-3}$ were chosen as the starting point for the recent studies. One important question is how to keep the global density at a roughly constant value during the phase with ECRH pulses, meaning that the density time traces shall, besides the modulation caused by ECRH, remain at a constant level. This implies that the density returns to its original value after each ECRH pulse, which is an essential requirement for testing the reproducibility of the effect. In general, keeping the density constant is achieved by feedbackcontrolling the gas inlet valves on the basis of the lineintegrated density measured by the central interferometer chord. This procedure allows to keep the density very well at the desired value during the flat-top phase of a discharge. When applying ECRH pulses that cause a modulation of the core density, however, secondary effects on the density result from operating the gas valves in feedback mode: A reduction of the core density causes an opening of the gas valves through the feedback loop, resulting in increased plasma fueling at the edge. As a result of this, there are two external factors acting on the plasma density, whose effects are difficult to disentangle in the further data analysis. Operating in gas feedback mode was found to speed up the flattening of the density profile by ECRH, as the reduction of the density in the center by the pump-out effect is accompanied by increased peripheral fueling which causes the edge density to rise. For the application of feedback controlling the shape of the density profile, operating in gas feedback mode was therefore convenient as it made the density response to central ECRH faster.

When studying the impact of local electron heating on the density profile, however, any modulation of the gas inlet rate in the phase with the ECRH pulses states an unacceptable biasing factor. It would prevent an unperturbed observation of the effect of ECRH, as also gas puff modulation affects the density profile. For this reason, the data set used for the study has to be restricted to those plasma discharges that were performed at a fixed gas inlet rate. This method is less convenient from the practical point of view, as keeping the density at the desired value then requires adequate fine-adjustment of the gas rate. The precise gas level that is required for staying at a certain density also depends on the machine condition, therefore, experience from previous discharges is necessary to get the right setting. However, only this method allows for unbiased observation of the ECRH impact on the density profile.

Besides modulation of the fueling rate, another biasing factor has to be taken into account: When performing experiments in L-mode discharges, the application of ECRH might increase the total heating power above the threshold for an L-H-transition. As this transition is characterized by the occurrence of an edge density pedestal, it has severe impact on the density profile. To sum up, an unbiased observation of the density response to central ECRH requires operation at a fixed gas fueling rate, and in case of L-mode experiments, it has to be made sure that the threshold for the transition to $\mathrm{H}$-mode is not reached when adding the desired level of ECRH power, e.g. by exploring the H-mode power threshold beforehand. In the following, only data from discharges will be used for deeper analysis in which these two points have been observed. Just to illustrate the possible impact of such biasing factors on the result, one example of a discharge will be shown where gas puff modulation due to feedback on the line-integrated density was present.

In all the experiments discussed in this article, the electron cyclotron heating system was operating at a frequency of $140 \mathrm{GHz}$, which corresponds to secondharmonic absorption at a toroidal field of 2.5 Tesla. To achieve central deposition of the heating power, it was therefore important to ensure that the magnetic axis is located close to the ECRH resonance layer and that the beam launcher is adjusted appropriately. By ray tracing calculations after the discharge, it has been verified that the ECRH power deposition is located at a normalized poloidal flux radius of not more than $\rho_{\text {pol }}=0.25$ in those discharges that are used for analysis.

The relevant quantities for analysis will be the electron temperature and density. A local measurement of the temperature can be obtained from the electron cyclotron emission (ECE) radiometer, which probes the plasma at 
points that are fixed in space for a given toroidal field. If the plasma moves or the flux surface geometry changes, the flux coordinate (like the normalized poloidal flux radius $\rho_{\text {pol }}$ ) corresponding to these points also changes. For this reason, time-dependent mapping of the ECE channels to flux radii is required. The density reconstruction in the plasma core relies on the line-integrated measurement by the five horizontal lines of sight of the DCN interferometer. The local densities that are required for this study are taken from a density profile, which is calculated by deconvolution of the line-integrated interferometric measurements and also includes data from lithium beam impact excitation spectroscopy in order to more reliably depict the density characteristics at the edge. This approach of integrated data analysis from multiple diagnostics, which is based on Bayesian probability theory, is described in detail in [7]. As discussed there, the uncertainties of the reconstructed density profile in the core region of the plasma are minimal at the radial locations where a chord of the interferometer is tangential to the corresponding flux surface. If the most central viewing chord of the interferometer does not intersect the magnetic axis, which is the case for the majority of plasma configurations on ASDEX Upgrade, the innermost part of the plasma is not probed by the interferometer and the density profile in this region has pure extrapolation character, resulting in very large error bars. In the further analysis, this innermost part of the plasma will therefore be excluded.

Figure 1 shows the time traces of a low-density L-mode discharge in which density pump-out is observed. For

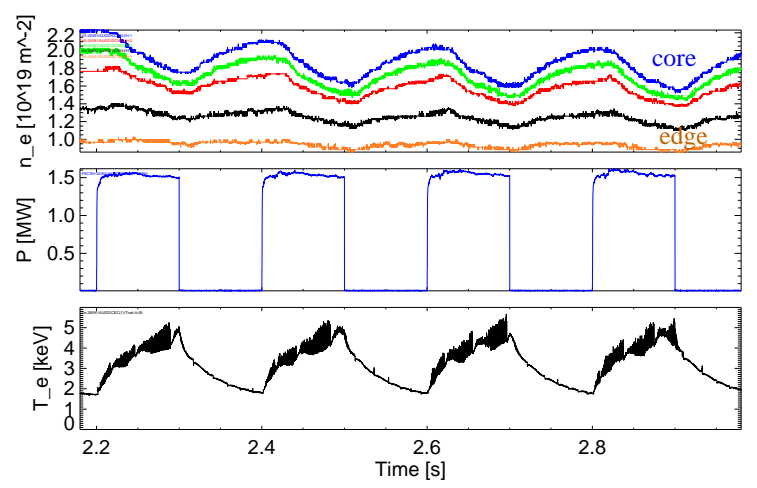

FIG. 1: Time traces of the 5 line-integrated densities measured by the DCN interferometer, applied ECRH power and electron temperature measured by an inner ECE channel (from top to bottom) for ASDEX Upgrade discharge \#25091 $\left(I_{p}=600 k A, B_{T}=2.5 T, q_{95}=6.3\right)$, in which density pumpout is observed. The energy confinement time in this discharge varies between roughly $220 \mathrm{~ms}$ (without ECRH) and $90 \mathrm{~ms}$ (with ECRH).

the ECRH, square wave power modulation with a period of $200 \mathrm{~ms}$ and $50 \%$ duty cycle is applied. As the
5 time traces of the DCN interferometer show, the density response to the heating pulses mainly occurs in the plasma center, whereas the edge interferometer channel does not show much modulation. The third time trace corresponds to a channel of the ECE radiometer, which probes the plasma at $\rho_{\text {pol }} \approx 0.3$. The pump-out effect in L-mode was found to be strong at low density only. Going towards higher density, it becomes weaker, then vanishes and finally reverses sign, i.e. above a certain density level, central heating increases the density in the plasma center. As we have already discussed in [6], the collisionality was found to be a good parameter for characterizing the density response to central heating. At low collisionality, the trapped electron mode (TEM) is the dominant microinstability and density pump-out is observed. Above a certain value of the collisionality, the ion temperature gradient (ITG) mode becomes the dominant turbulence type and central heating peaks the density profile [8]. This is consistent with the observations presented in [9], according to which thermodiffusion reverses sign at the transition from TEM to ITG. In H-mode discharges, we found that for a wide range of discharge parameters, no significant density response to central ECRH can be observed. This is regularly the case when the applied neutral beam injection (NBI) power, which is used to obtain $\mathrm{H}$-mode, is much higher than the available ECRH power. At least one set of parameters at low plasma current $\left(I_{p}=600 k A\right)$ was discovered for which clear density modulation can be observed. The corresponding time traces of a discharge that was heated by $2.5 \mathrm{MW}$ of NBI are presented in figure 2 . Here, heating in the core peaks

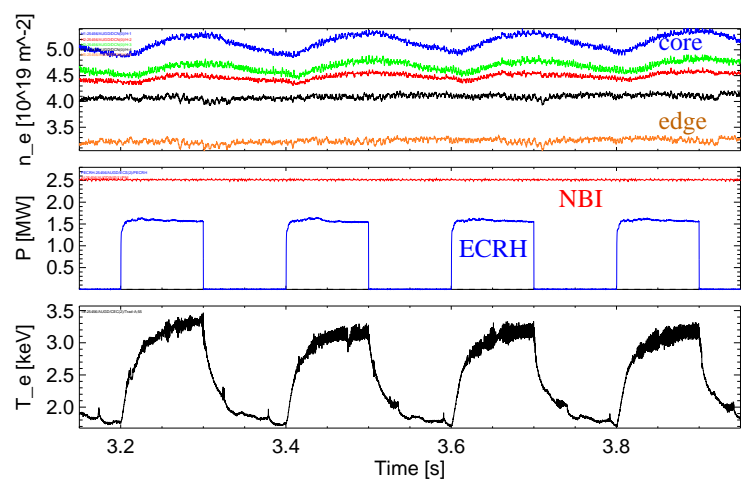

FIG. 2: Time traces of the 5 line-integrated densities measured by the DCN interferometer, applied ECRH and NBI power and electron temperature measured by an inner ECE channel (from top to bottom) for ASDEX Upgrade discharge \#25456 $\left(I_{p}=600 k A, B_{T}=2.5 T, q_{95}=6.6\right)$. In this H-mode discharge, central heating increases the core density. The energy confinement time in this discharge is about $65 \mathrm{~ms}$.

the density profile, as can be seen from the behavior of the core interferometer channel. The edge channels, in contrast, do not show any modulation. Similar ASDEX 
Upgrade discharges that show identical behavior are discussed in [8]. The detailed transient analysis of the discharges shown in figures 1 and 2 is presented in the following section.

\section{TRANSIENT BEHAVIOR OF LOCAL TEMPERATURE AND DENSITY}

The aim of this study is to monitor the temporal evolution of the local electron temperature and density at a given location in the plasma during the ECRH modulation phase. In the experiments discussed here, the minimum flux radius down to which the ECE diagnostic probes the plasma is slightly inside $\rho_{\text {pol }}=0.2$, which restricts the radial range that can be used for analysis. A more stringent limitation, however, results from the presence of sawtooth activity. This is illustrated in figure 3, which shows time traces for three different ECE channels in an L-mode discharge. The signal of the inner-

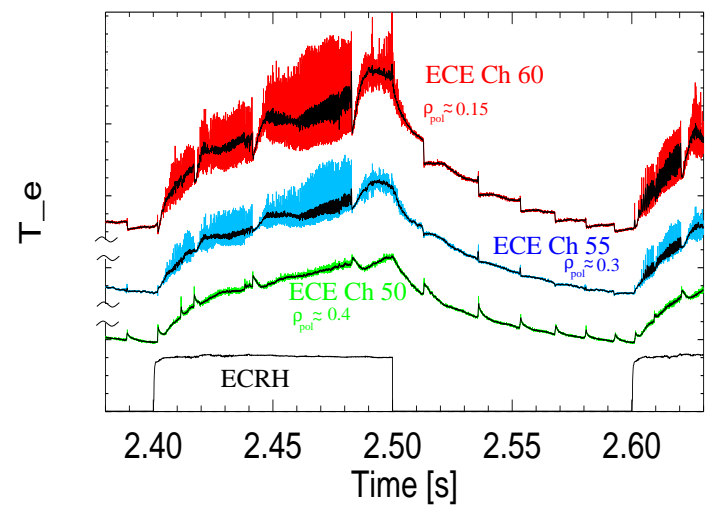

FIG. 3: Time traces of 3 ECE channels for discharge \#25091. The time traces are shown with an artificial vertical offset for better visibility. The ordinate is scaled in $2 \mathrm{keV}$ per (large) division, but no absolute scale is given due to the offsets. The 3 black time traces show the result of averaging the signals over 0.3 milliseconds. Channel 60 probes the plasma at $\rho_{\text {pol }} \approx 0.15$, channel 55 at $\rho_{\text {pol }} \approx 0.3$ and channel 50 at $\rho_{\text {pol }} \approx 0.4$. For reference, the applied ECRH power is shown by the bottom trace (in arbitrary units).

most ECE channel is dominated by precursor oscillations with a frequency around $3 \mathrm{kHz}$ in the phases with ECRH on, which are temporarily interrupted each time a sawtooth crash occurs. In the phases without ECRH, there are no precursor oscillations, but each crash causes a sudden drop of temperature. Further outwards, the steps in temperature in the ECRH off phase vanish, the crashes just appear as short spikes on the time trace. The precursor oscillations in the ECRH heated phase, however, remain. Even further outside, also the precursors disappear. Figure 4 shows a spectrogram of the ECE data at $\rho_{\text {pol }} \approx 0.3$. The precursor oscillations in the ECRH

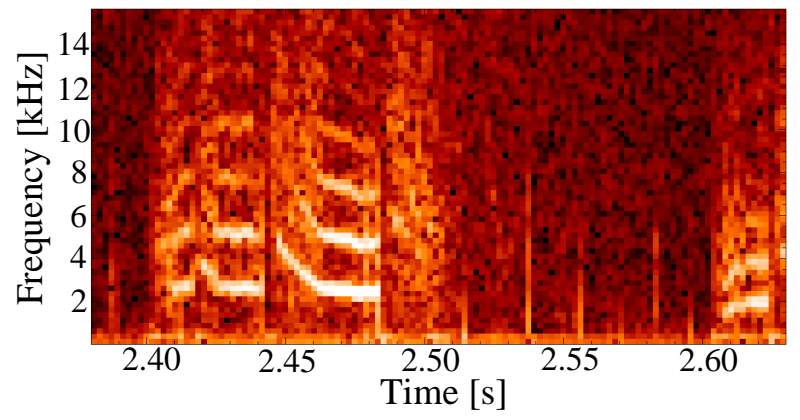

FIG. 4: Spectrogram of ECE channel $55\left(\rho_{\text {pol }} \approx 0.3\right)$ for discharge \#25091. The sawtooth precursor oscillations at about $3 \mathrm{kHz}$, as well as higher harmonics, are clearly visible.

phase are clearly visible and have a frequency of about 3 $\mathrm{kHz}$, including also higher harmonics. This sawtooth behavior seems to be similar to the observations presented in [10], where ECRH deposition close to the $q=1$ surface has been shown to strongly influence the sawtooth characteristics. All in all, the occurrence of sawtooth activity has two consequences for our data analysis, which includes $T_{e}$ measurements: On the one hand, ECE data from the innermost channels $\left(\rho_{p o l}<0.25\right)$ should not be used, as the sudden drops of temperature caused by sawtooth crashes would then bias the result. Outside $\rho_{\text {pol }}=0.25$, these crashes just cause short spikes on the temperature signal, which will result in single outliers in the following scatter plots, but not distort them. On the other hand, the fast sawtooth precursor oscillations would considerably increase the scatter in the following density-temperature diagrams when analyzing a radial interval of about $0.25 \leq \rho_{\text {pol }}<0.4$. For this reason, the ECE data will be averaged over time intervals of $0.3 \mathrm{~ms}$ in order to reduce the scatter caused by the precursor oscillations. The smoothed ECE time traces obtained that way are also shown in the figure. In the phase without $\mathrm{ECRH}$, the effect of averaging is marginal.

When extracting local densities from the density profile, which relies on interferometric measurements in the plasma center, one has to be aware that the innermost channel of the interferometer reaches a minimum $\rho_{\text {pol }}$ of about 0.2 in the given discharge, but does not penetrate deeper towards the plasma center. For this reason, the density profile has just extrapolation character in the region $0 \leq \rho_{\text {pol }}<0.2$, but is not based on a measurement. As discussed above, the use of ECE data inside $\rho_{\text {pol }}=0.25$ is unfavorable anyway, therefore, from this property of the density measurement, no additional restriction of the usable radial range for analysis results. In contrast to the local ECE measurement, the lineintegrated measurement by the interferometer is hardly affected by the sawtooth activity in the plasma center, as 
the smooth density time traces in figure 1 illustrate. The diagnostic lithium beam sent into the plasma is chopped for technical reasons [11], with a period of typically $80 \mathrm{~ms}$ and an off-time of $24 \mathrm{~ms}$. For this reason, there are periodically occurring intervals of $24 \mathrm{~ms}$ in which the density profile is not available. As we always inject a series of identical ECRH pulses into the plasma and the ECRH modulation period $(200 \mathrm{~ms})$ is not an integer multiple of the lithium beam chopping period of $80 \mathrm{~ms}$, these gaps in the time base of the density measurement do not hinder the analysis.

For the analysis, a series of ECRH pulses is selected which were injected into a plasma with constant global parameters. Then, the temperature and density data at the radial location of interest is extracted from the diagnostics mentioned above. As the time resolution of the diagnostics differs, the data points are mapped onto a common, coarser time base. The result is then visualized as a scatter plot in which the local density is plotted versus the local temperature. As an example of this, we discuss an NBI-heated H-mode discharge in which the addition of central ECRH increases the peaking of the density profile. The corresponding time traces of heating power, line-integrated density and electron temperature have already been presented in figure 2. The radial profiles of temperature and density, which occur at the end of the ECRH-on and ECRH-off phase, respectively, are shown in figure 5. It can be seen that the density response to central heating is, in this case, restricted to the inner part of the plasma. Outside $\rho_{\text {pol }}=0.6$, there is no significant change of density. The radial range of interest for creating density-temperature diagrams is therefore narrow, $\rho_{\text {pol }}$ must not be smaller than 0.25 due to the lack of reliable ECE data further inside, but should be clearly below 0.4 in order to have substantial density modulation. Figure 6 shows the case $\rho_{\text {pol }}=0.25$. The bottom left corner in the diagram corresponds to the case where ECRH has been off for $100 \mathrm{~ms}$ and is just being switched on again, and the top right corner to the case where ECRH has been on for $100 \mathrm{~ms}$. The remarkable observation is the fact that the trajectory in the density-temperature diagram along which the system evolves between those two points is not a straight line. In contrast, a hysteresis loop is observed which is run through in the counterclockwise direction. To illustrate the temporal order, the data points corresponding to 4 different phases of the ECRH cycle are shown with different symbols. The first and the second $50 \mathrm{~ms}$ of the ECRH phase as well as the first and second $50 \mathrm{~ms}$ of the phase without ECRH can be distinguished that way. When ECRH is switched on, there is first a rapid response of temperature, whereas density remains initially constant. Accordingly, starting from the bottom left corner of the curve, the trajectory first goes to the right, parallel to the temperature axis. Only with some delay, density starts increasing and the trajectory becomes curved. Finally, the point in the top right corner is reached. When ECRH is switched off, temperature again responds faster and decreases imme-
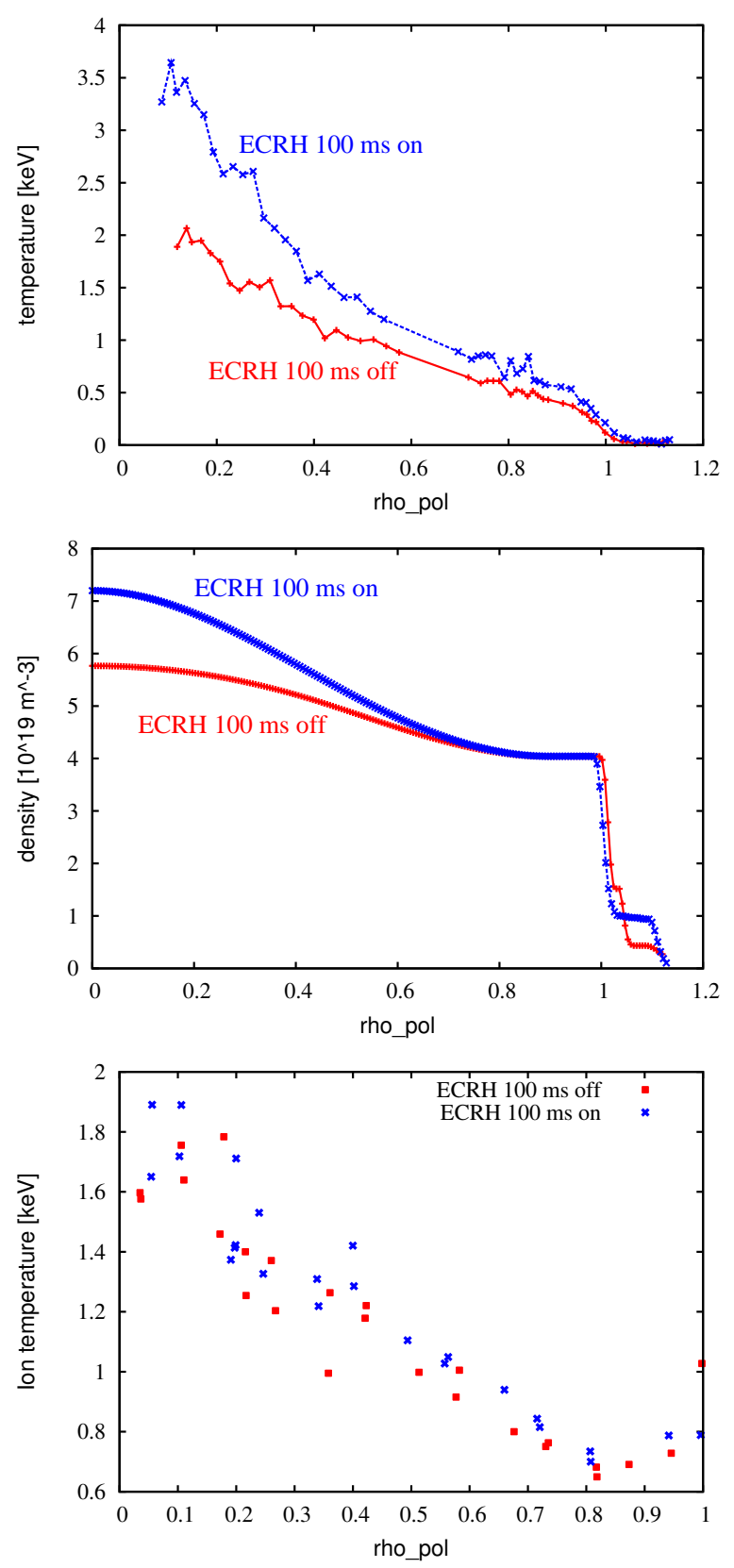

FIG. 5: Radial profiles of electron temperature (top) and density (center) and ion temperature (bottom) for ASDEX Upgrade discharge \#25456. The two profiles in each plot panel correspond to the case that ECRH has been off or on for 100 $\mathrm{ms}$, respectively. They correspond to time index $t=3.4 \mathrm{~s}$ and $t=3.5 \mathrm{~s}$ (compare figure 2).

diately, whereas the density response occurs with delay. The result is a hysteresis curve. It is worth mentioning that this curve is run through several times, corresponding to the number of ECRH pulses, and the data points from different pulses overlap well, which underlines the reproducibility of the effect.

The occurrence of such hysteresis loop is not a pecu- 


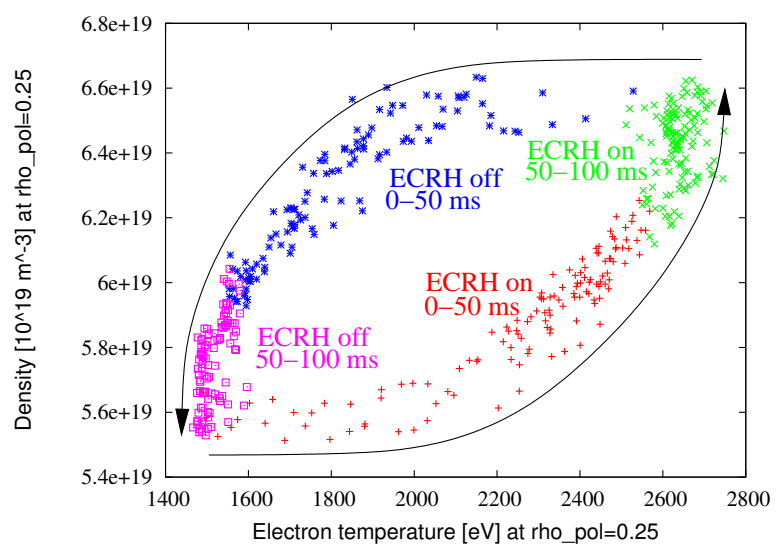

FIG. 6: Density-temperature diagram at $\rho_{\text {pol }}=0.25$ for ASDEX Upgrade discharge \#25456 during the ECRH modulation phase. Data corresponding to four different time intervals with respect to the beginning of the heating pulse are shown with different symbols (first $50 \mathrm{~ms}$ of ECRH pulse, second 50 $\mathrm{ms}$, first $50 \mathrm{~ms}$ after pulse and $50-100 \mathrm{~ms}$ after pulse). The curve is run through in the counter-clockwise direction.

liarity of H-mode discharges, it was observed in practically all our experiments in which a density response to central heating was present. A further example, namely a low-density L-mode discharge whose time traces were presented in figure 1 already, is shown in figures 7 and 8. Here, density pump-out is observed. As the density profiles in figure 7 illustrate, the pump-out effect affects the whole radial range from the core to the edge, in contrast to the previously discussed discharge where only the core plasma showed density modulation. Accordingly, it is here possible to plot density-temperature diagrams for a wider range of flux radii, see figure 8 . In this case, where heating causes a flattening of the density profile, the points where ECRH is switched on and off are located at the top left and bottom right corner of the diagram, and the hysteresis loop is run through in the clockwise direction. As the different radial positions in figure 8 show, the behavior is qualitatively the same over a wide range, just the absolute values of temperature and density and the amplitude of variation decreases towards the edge. In the two cases discussed so far, the gas inlet valves were controlled in feed-forward mode, and in the L-mode case, the applied ECRH power was far below the L-H threshold. Under these conditions, we always observe hysteresis curves of that kind, i.e. curves that are characterized by fast response of temperature and slower response of density. Just to illustrate the potential bias that occurs when these two rules are neglected, we show two examples in figure 9. The left diagram shows an L-mode discharge in which density pump-out occurs, but the gas inlet valves were feedback controlled by the core interferometer channel. As ECRH is switched on and core density decreases, additional gas puff at the edge occurs. As a result of this, the hysteresis curve close to the edge
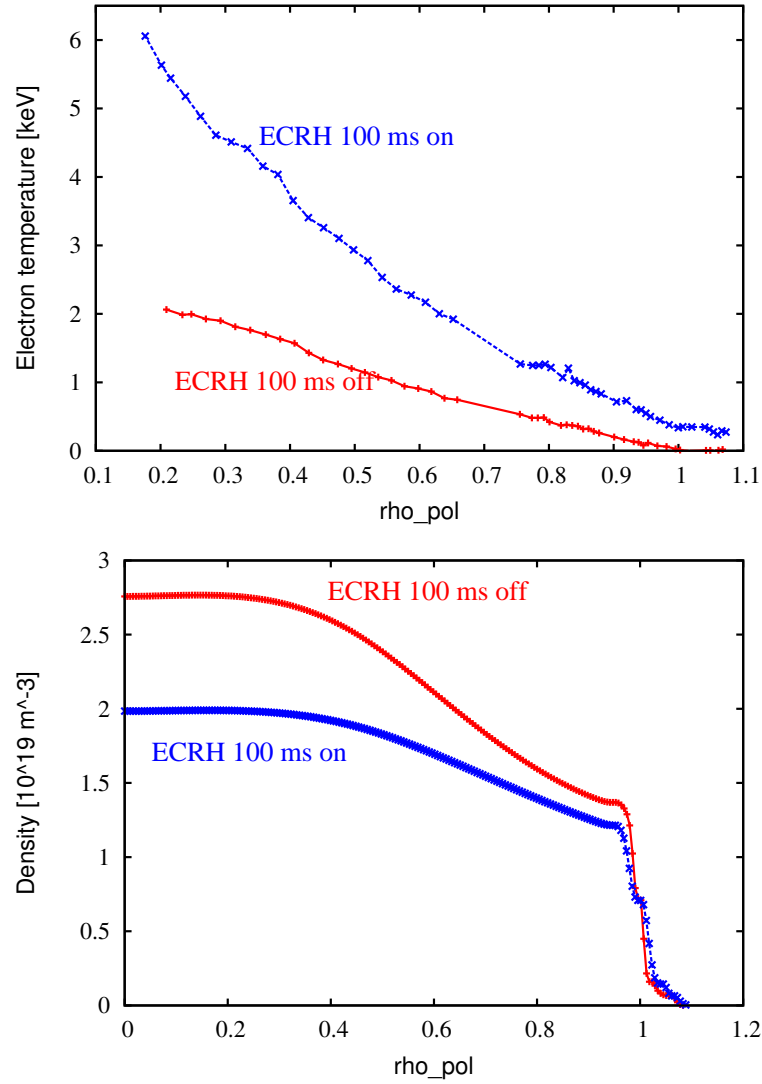

FIG. 7: Radial profiles of temperature (top) and density (bottom) for ASDEX Upgrade discharge \#25091. The two profiles in each plot panel correspond to the case that ECRH has been off or on for $100 \mathrm{~ms}$, respectively. They correspond to time index $t=2.6 \mathrm{~s}$ and $t=2.7 \mathrm{~s}$ (compare figure 1 ).

$\left(\rho_{\text {pol }}=0.9\right)$ has an unusual shape and is run through in the counter-clockwise direction. The curves in the center and at mid-radius are still run through clockwise, which is characteristic of density pump-out, but their shape is distorted. When ECRH is turned on, they initially start in the usual manner, but as the additional gas puffed at the edge propagates to the center, the decrease of density stops and especially the curve at $\rho_{\text {pol }}=0.25$ develops a tail that is pointing to the right. The area enclosed by the hysteresis loops is strongly reduced that way. The right diagram in figure 9 corresponds to an Lmode discharge with higher density, where central heating increases the core density. In this discharge, the gas inlet valves were also feedback-controlled, but in addition, the ECRH power was high enough to trigger an $\mathrm{L}-\mathrm{H}$ transition. Accordingly, the observation of the density response to central heating is biased by two factors. Close to the edge, a hysteresis loop is observed which is run through in the counter-clockwise direction. Further in the center, however, where a clear increase of density was observed when applying pulsed ECRH with reduced power, no loop is present any more. The overall behavior 


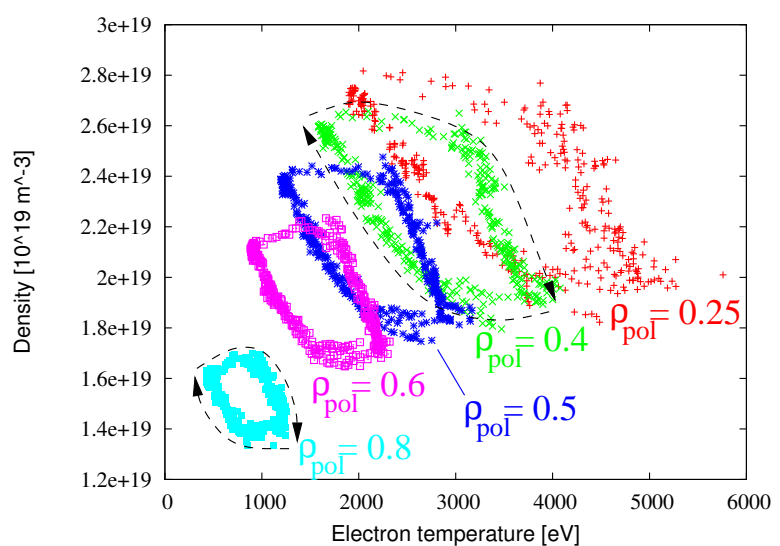

FIG. 8: Density-temperature diagrams at five different radii for ASDEX Upgrade discharge \#25091 during the ECRH modulation phase. All the curves run through in clockwise direction, as indicated by arrows for two cases.

in the center (higher temperature corresponds to lower density) is even opposite to the response at lower ECRH power. Detailed analysis of this discharge revealed that roughly in the middle of an ECRH pulse, the plasma has accumulated sufficient energy to enter into a weakly developed H-mode, which strongly affects the density profile. The transition back to L-mode occurs roughly 50 ms after the end of the heating pulse. As a result of this, the minima and maxima of the core density do no longer coincide with the beginning and the end of the heating pulses, but with the L-H and H-L transitions, which occur about $50 \mathrm{~ms}$ later. Accordingly, the basic effect of central ECRH on the density profile is overlaid by the stronger impact of $\mathrm{L}-\mathrm{H}$ and $\mathrm{H}-\mathrm{L}$ transitions in this discharge.

These examples underline that much care has to be taken in the experiments in order to observe the density response to central heating in an unbiased way. In general, this task is easier to fulfill in H-mode discharges, as the triggering of a biasing L-H transition is then excluded per se. In any case, the gas inlet rate has to be kept fixed during the ECRH modulation phase. The proper adjustment of this rate, which guarantees the obtainment of the desired global density, requires fine-tuning and possibly some trial and error work.

\section{TRANSPORT SIMULATIONS WITH THE ASTRA CODE}

The observation of hysteresis loops in the local densitytemperature diagram in experiments with pulsed central ECRH has, to our knowledge, not been reported in literature before. For us, the question arises whether the occurrence of such loops can be reproduced by an empirical, preferably simple transport model. Therefore, numerical simulations with the ASTRA code [12] have
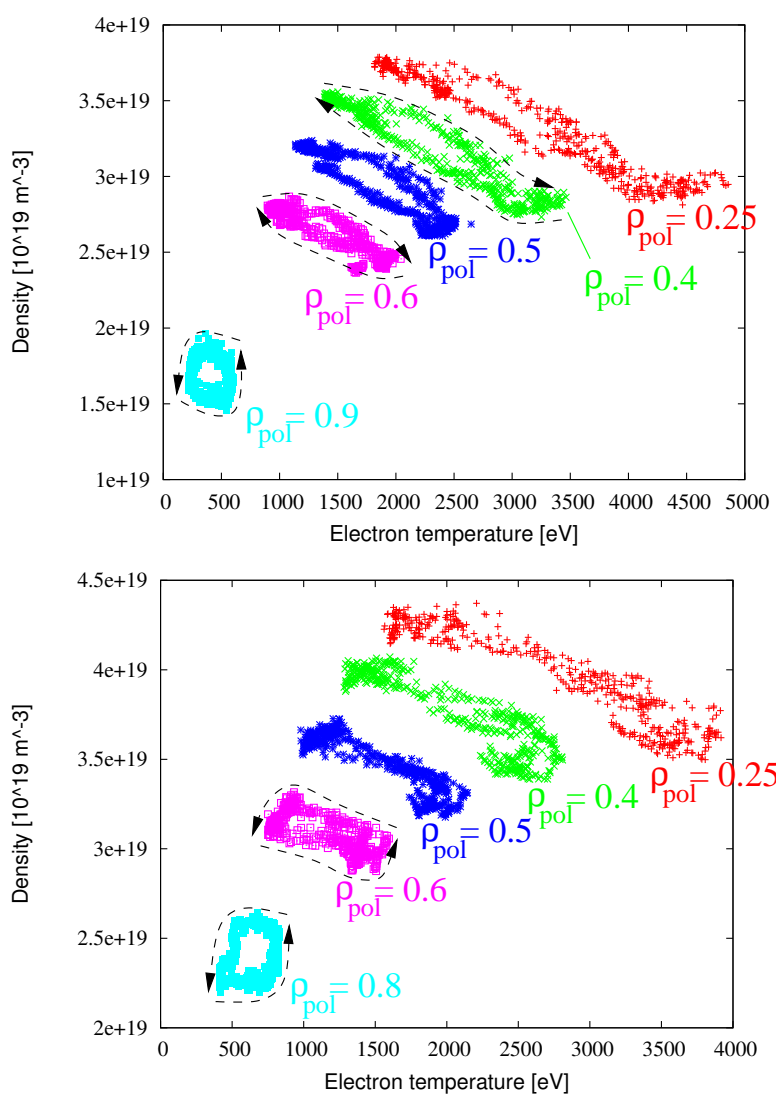

FIG. 9: Two examples of density-temperature diagrams which are observed when biasing factors are present. Left: The gas inlet valves were feedback controlled by the core interferometer channel. Accordingly, density pump-out in the center causes increased fueling from the edge. As a consequence of this, the hysteresis loop at $\rho_{\text {pol }}=0.9$ has different shape and is run through in the counter-clockwise direction (see arrows). Right: Gas valves were feedback controlled, and in addition, the ECRH power is high enough to trigger an L-H transition.

been performed. The simulation is based on the following equations for the heat flux $\vec{q}_{e}$ and the particle flux $\vec{\Gamma}_{e}$ :

$$
\begin{gathered}
\vec{q}_{e}=-\chi_{e} n_{e} \vec{\nabla} T_{e} \\
\vec{\Gamma}_{e}=-D \vec{\nabla} n_{e}-D_{T} n_{e} \frac{\vec{\nabla} T_{e}}{T_{e}}+\vec{v}_{p} n_{e}
\end{gathered}
$$

In equation (1), we assume pure diffusion for the electron heat transport and label the corresponding transport coefficient as $\chi_{e}$. In equation (2), the labeling of the coefficients follows the definition in [13], where $D$ stands for the particle diffusion coefficient, $D_{T}$ is the thermodiffusion coefficient, which provides coupling between density and temperature profile, and $v_{p}$ is the inward pinch term, which allows for the occurrence of centrally peaked density profiles also in the absence of particle sources in the 
plasma center.

In the ASTRA calculations, a fixed ion temperature profile is assumed, which was obtained by a charge exchange spectroscopy measurement in the corresponding experiment. It can be seen in figure 5 that the ion temperature does not change significantly in the ECRH modulation phase. For electron temperature and density, the measured profiles serve as initial condition only. These two quantities are then evolved in time according to the fluxes given by the above transport equations. The transport equations are solved inside a normalized toroidal flux radius $\rho_{\text {tor }}$ (which is the natural coordinate in ASTRA and will therefore be used in the following discussion) of 0.9 . At this radius, the experimental data serves as boundary condition. That way, there is no need to model the edge pedestal characteristics.

For the heat transport coefficient $\chi_{e}$, we use the critical gradient model described in [14], which has been successfully applied to electron heat transport studies on ASDEX Upgrade in the past. The free parameters in this model are adjusted in a way to provide consistency between simulated and measured temperature profiles. They are assumed not to be time-dependent, i.e. they do not change from the ECRH on to the ECRH off phase. The settings made in the present work are similar to those used in previous studies, e.g. [15]. For the threshold, the formula given in [16] is used, which has been shown to yield good results in [15]. When performing the parameter adjustment, we only take into account the two temperature profiles that occur after $100 \mathrm{~ms}$ ECRH on and $100 \mathrm{~ms}$ ECRH off, namely those shown in figure 5 .

The challenge then is to adjust the particle transport coefficients $D, D_{T}$ and $v_{p}$ in equation (2) in a way to provide consistency between the simulated and the measured density profiles. Possibly, several sets of coefficients exist which reproduce the density profiles after $100 \mathrm{~ms}$ ECRH on and $100 \mathrm{~ms}$ ECRH off well, but differ in view of the temporal evolution, i.e. yield different hysteresis loops. In the following, several approaches to coefficient selection will be presented.

To provide a coupling between density and temperature, the usual approach would be to make use of the thermodiffusion term with coefficient $D_{T}$. As this term is proportional to the normalized gradient of the electron temperature, $\vec{\nabla} T_{e} / T_{e}$, we have extracted this quantity from the temperature profile delivered by the critical gradient model for two points in time, after $100 \mathrm{~ms}$ ECRH off and $100 \mathrm{~ms}$ ECRH on. It was found that $\vec{\nabla} T_{e} / T_{e}$ changes in the following way when ECRH is switched on: It decreases inside of the ECRH deposition radius and outside $\rho_{\text {tor }} \approx 0.5$, but increases in between. The density profile, in contrast, peaks in the region from the magnetic axis to $\rho_{\text {tor }} \approx 0.4$ and does hardly change further outside. This different radial behavior makes it difficult to explain the density response on the basis of thermodiffusion. Within several attempts, which also included a radial dependence of $D_{T}$, no set of transport coefficients was found which couples density and temperature via thermodiffusion and reproduces the experimentally observed density profiles well.

An alternative way of providing a coupling between density and temperature profile is to assume that the particle diffusion coefficient $D$ is proportional to $\chi_{e}$. As the application of central heating increases the heat diffusion coefficient according to the critical gradient model, also $D$ increases and changes the density profile. This approach might work in cases where central heating causes density pump-out, as an increase of $D$ flattens the density profile. In cases where density peaking in response to central ECRH is observed, like in discharge \#25456, however, a decrease of $D$ is required to explain the density response. Accordingly, the proportionality factor between $D$ and $\chi_{e}$ would have to be negative. As the diffusion coefficient $D$ must not be negative, however, this approach is not useful for discharges where central heating peaks the density profile.

Empirically, it was finally found that rather realistic results can be obtained by setting the thermodiffusion coefficient $D_{T}$ to zero and performing the coupling between density and temperature by giving the inward pinch term $v_{p}$ a temperature dependence. This ansatz is motivated by the recent findings presented in [8], which indicate that a correlation exists between the electron to ion temperature ratio $T_{e} / T_{i}$ and the density gradient length, and accordingly also the peakedness of the density profile. From the practical point of view, this approach is very promising as the radial dependence of $T_{e} / T_{i}$ (which can easily be seen from figure 5 as $T_{i}$ hardly varies) is similar to that of the density: It increases from the plasma center to mid-radius when ECRH is switched on, but hardly changes further outside. Hence, we use:

$$
\begin{gathered}
D_{T}=0 \\
v_{p}\left(\rho_{\text {tor }}, t\right)=v_{\text {Ware }}\left(\rho_{\text {tor }}, t\right)+v_{1} \cdot f_{1}\left(\rho_{\text {tor }}\right)+ \\
+v_{2} \cdot f_{2}\left(\rho_{\text {tor }}\right) \cdot \frac{T_{e}\left(\rho_{\text {tor }}, t\right)}{T_{i}\left(\rho_{\text {tor }}\right)}
\end{gathered}
$$

Here, $v_{W a r e}$ is the neoclassical Ware pinch [17], which we include in our model, and $f_{1}$ and $f_{2}$ are radial functions which have to fulfill the condition $f_{1}(0)=f_{2}(0)=0$. This is necessary as $v_{p}$ has to go to zero in the limit $\rho \rightarrow 0$. Otherwise, the model would deliver a non-zero density gradient at the magnetic axis, resulting in density profiles with non-zero derivative in the plasma center. The choice of $f_{1}$ and $f_{2}$ gives us additional freedom to match the simulated profiles to the experimentally observed ones. Figure 10 shows the graph of $f_{1}$ and $f_{2}$ that we use in the simulation. Both functions are polynomials which are obtained by multiplying terms like $(1-\rho)^{M}$ and $\left(1-(1-\rho)^{N}\right)$, where $M$ and $N$ are positive integer numbers. These terms ensure that both functions go to zero in the limit $\rho \rightarrow 1$ and $\rho \rightarrow 0$, respectively. For both functions, $N$ is greater than $M$ in 


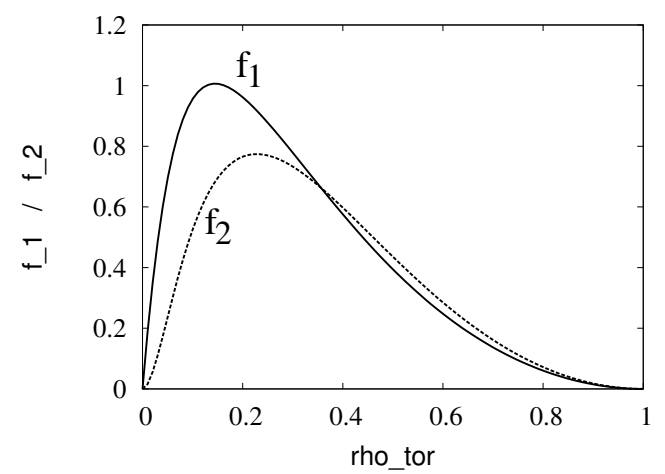

FIG. 10: Graph of the radial functions $f_{1}\left(\rho_{\text {tor }}\right)$ and $f_{2}\left(\rho_{\text {tor }}\right)$ used in the simulation.

order to have a steep drop to zero in the center and a slower decay to zero towards the edge.

For the particle diffusion coefficient $D$ and the coefficients $v_{1}$ and $v_{2}$, we use static values which are constant in time and radius and accordingly do not depend on temperature, density and the respective gradients. By adjusting the ratio of $D, v_{1}$ and $v_{2}$, it was possible to match the two simulated density profiles occurring after $100 \mathrm{~ms}$ with ECRH on and $100 \mathrm{~ms}$ with ECRH off to the measured profiles. This is illustrated in figure 11, which compares the simulated temperature and density profiles for an NBI-heated H-mode discharge to those that were experimentally observed in ASDEX Upgrade discharge \#25456. We take into account the fueling profile of the neutral beam heating in our model. As central heating increases the core density in this discharge, $v_{2}$ has negative sign. $v_{1}$, in contrast, is positive and provides an outward-directed flux which is independent of temperature and counteracts the term with $v_{2}$. The fact that density changes mainly in the center when ECRH is applied is reflected by the properties of $f_{1}$ and $f_{2}$, which mainly differ for $\rho_{\text {tor }}<0.4$, but have very similar shape further outside. When ECRH is switched on and $T_{e} / T_{i}$ increases, this results in increasing density in the center. Figure 12 illustrates the effect of setting $v_{1}$ to zero and varying $v_{2}$. A low (absolute) value for $v_{2}$ results in a realistic density profile for the case without ECRH, but does not provide enough density modulation. With a higher absolute value for $v_{2}$, density modulation is realistic, but the density profiles are much too peaked. For this reason, the additional, outward-directed term with positive coefficient $v_{1}$ is required to obtain a realistic description of the density profile. It is worth mentioning that up to this point, only two points in time, just before the beginning and at the end of an ECRH pulse, have been used as reference for adjusting the model parameters.

Once the model has been set up this way, it is possible to extract the temporal evolution of the local temperature and density from the simulation and generate a
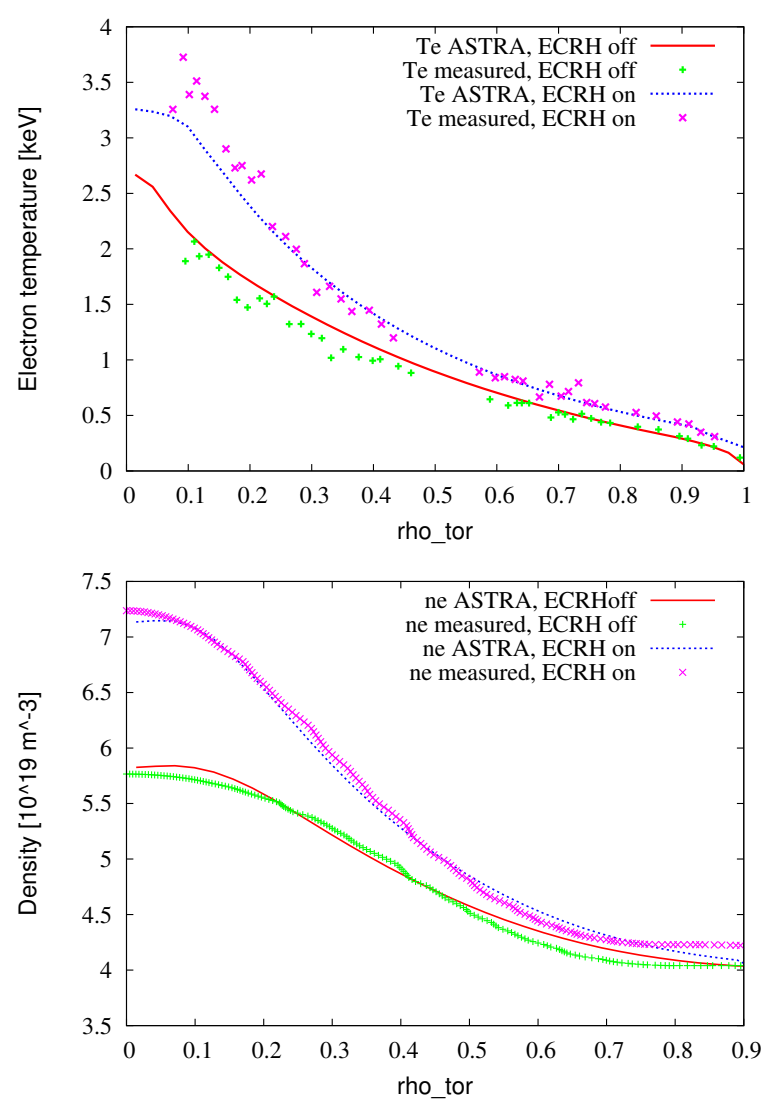

FIG. 11: Comparison of the profiles simulated by the ASTRA model (solid / dashed lines) to measured profiles (points, discharge \#25456). The left diagram shows the temperature profiles and the right diagram the density profiles. The two profiles in each plot panel correspond to the cases where ECRH was on and off for $100 \mathrm{~ms}$, respectively. The settings used for this simulation are $D=2.0 \frac{\mathrm{m}^{2}}{\mathrm{~s}}, v_{1}=2.0 \frac{\mathrm{m}}{\mathrm{s}}$ and $v_{2}=-4.4 \frac{\mathrm{m}}{\mathrm{s}}$. The abscissa gives $\rho_{\text {tor }}$, which is the preferred radial coordinate for the ASTRA model.

density-temperature diagram, as it was done for the experimental data. The result is shown in figure 13 . Evidently, the simulation yields a hysteresis curve which is run through in the counter-clockwise direction. Like the experimental curve, it is characterized by a fast response of the temperature, but a delayed response of the density when the heating power level is altered. The shape of the curve sensitively depends of the ratio $D / \chi_{e}$, as illustrated in the figure. When $D$ is altered, but $\chi_{e}$ and the ratios $v_{1} / D$ and $v_{2} / D$ are kept fixed, the profiles shown in figure 11 do not change much, unless the values of $D, v_{1}$ and $v_{2}$ are so low that the Ware pinch starts playing a significant role. The speed with which the density responds to changes of the temperature varies with $D / \chi_{e}$. At low values, the hysteresis curve is almost parallel to the density axis at the end of a heating pulse, meaning that the temperature has already reached a static level, whereas the density is still evolving. Going 

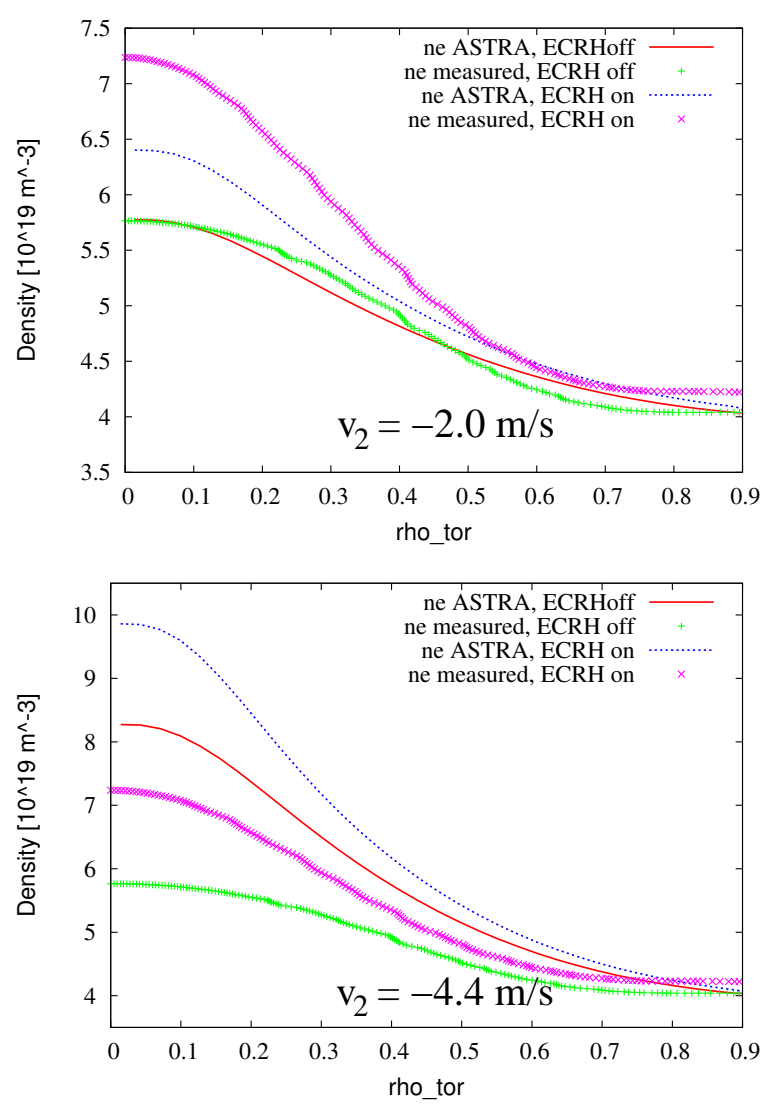

FIG. 12: Effect of the variation of the coefficient $v_{2}$. The diagrams show the resulting density profile like in the right plot panel of figure 11, but with $v_{1}=0$. In the left diagram, $v_{2}$ is set to $-2.0 \frac{\mathrm{m}}{\mathrm{s}}$. This yields a realistic density profile when ECRH is off, but the increase of density in the center is too low when ECRH is switched on. In the right diagram, $v_{2}$ is set to $-4.4 \frac{\mathrm{m}}{\mathrm{s}}$, which results in the appropriate amount of density modulation in the center, but yields absolute densities that are much too high.

to higher values of $D / \chi_{e}$ speeds up the density response and the shape of the hysteresis loop changes. Comparison with the experimental result (figure 6) shows that reasonable agreement can be achieved by setting $D$ to a value around $2 \frac{m^{2}}{s}$, which results in $D / \chi_{e} \approx 1$ at the radial location shown in the figure when ECRH is on. Having a look at the bottom left and top right corners of the curves in figure 13, it can be seen that the amplitude by which the temperature varies is almost the same in all three cases. The amplitude of density modulation is similar for $D=2.0 \frac{\mathrm{m}^{2}}{\mathrm{~s}}$ and $D=4.0 \frac{\mathrm{m}^{2}}{\mathrm{~s}}$, which means that a stationary state is reached within the $100 \mathrm{~ms}$ of an ECRH pulse. In the case $D=1.0 \frac{\mathrm{m}^{2}}{\mathrm{~s}}$, however, the amplitude of density modulation is clearly reduced. In this case, density response is so slow that the stationary state is not reached within the given time. The vertical offset of the curves is due to the influence of the Ware pinch, which makes the profiles the more peaked the

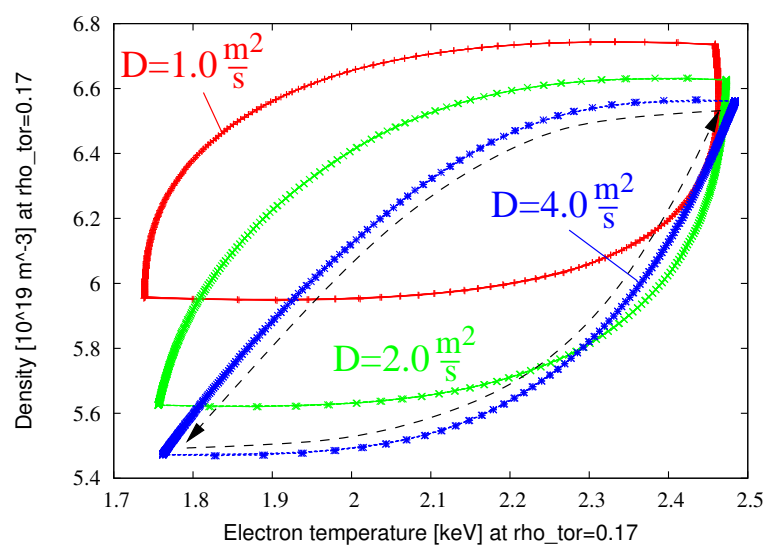

FIG. 13: Local density-temperature diagram at $\rho_{\text {tor }}=0.17$ (corresponding to $\rho_{\text {pol }} \approx 0.25$ ) resulting from the ASTRA simulation. The curves are run through in the counterclockwise direction, as indicated by the dashed arrows. The effect of varying the value of the diffusion coefficient $D$, keeping the ratios $v_{1} / D$ and $v_{2} / D$ constant, is illustrated by the three different curves.

lower the diffusion coefficient $D$ is.

The setting $D=2.0 \frac{m^{2}}{s}$ provides best agreement between the shape of the simulated and the measured hysteresis curve for discharge \#25456. Figure 14 shows both curves at the radial location $\rho_{\text {tor }}=0.17$. As the diagrams in figure 11 have shown, maximum and minimum density agree very well, whereas the amplitude of temperature modulation at $\rho_{\text {tor }}=0.17$ is lower in the simulation. In the left plot panel of figure 11, it can be seen that at this radius, the ECE measurement is above the simulated temperature in the ECRH on case, and below it in the ECRH off case. When going radially outward, simulated and measured electron temperatures match better.

From the ASTRA simulations made so far, we draw

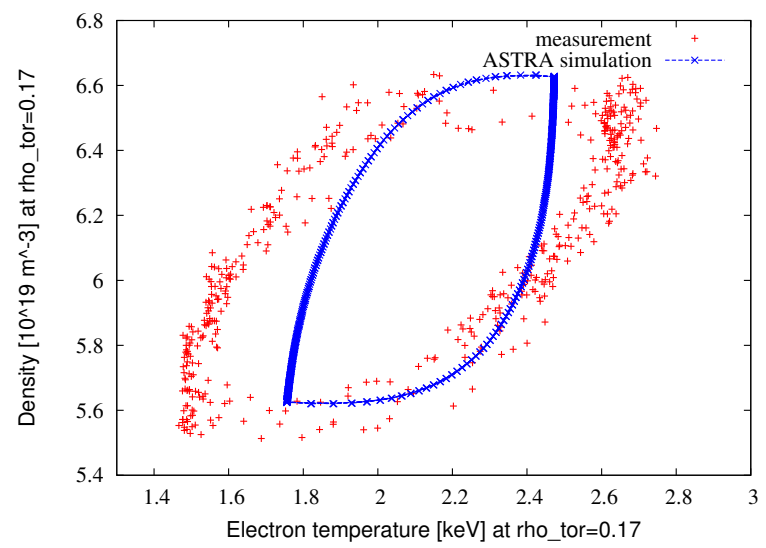

FIG. 14: Comparison of the experimental hysteresis curve (figure 6) and the simulated curve for $D=2.0 \frac{\mathrm{m}^{2}}{\mathrm{~s}}$ (figure 13). 
the following conclusions:

When studying the density response to central heating in tokamak plasmas, the analysis of the transient behavior of density and temperature provides additional information on the underlying transport processes. Just by looking at the results obtained directly before and at the end of a heating pulse, i.e. after some settling time, one could only determine the ratios $v_{1} / D$ and $v_{2} / D$ in our model. Only a look at the transient behavior, namely the trajectory in the local density-temperature diagram, however, can also provide information on $D / \chi_{e}$. Therefore, temporally resolved analysis has the potential of delivering further constraints on transport models and on the coefficients and their interdependences within those models, as already known for other types of transport studies [18].

In our present, rather basic approach with the ASTRA code, the model parameters have been adjusted by hand in an iterative way until the desired level of agreement between simulated and measured temperature and density profiles was achieved. As this is very time-consuming, only a limited number of parameter sets could be tested. We found that by neglecting the thermodiffusion term and introducing a temperaturedependent inward pinch, realistic simulation results can be achieved. However, we so far cannot exclude that a set of model parameters with non-zero thermodiffusion and temperature-independent $v_{p}$ exists which describes the experimental observations just as well. Therefore, we avoid drawing conclusions on the temperaturedependence of the pinch at the moment.

We are planning to perform model parameter adjustment by a computer algorithm in the near future. Once a transport model with a certain number of free parameters has been defined in the ASTRA code, it makes a prediction of the temporal evolution of the temperature and density profile, $T_{e, A S T R A}(\rho, t)$ and $n_{e, A S T R A}(\rho, t)$. By comparing them to the experimental values $T_{e}(\rho, t)$ and $n_{e}(\rho, t)$ on a discrete, 2-dimensional mesh (corresponding to time and radius), a kind of mean square deviation between simulation and measurement can be defined, which takes into account the full temporal evolution of the profiles. By minimizing this mean square deviation, a computer algorithm can then deliver the set of transport coefficients which best reproduces the experimental result. As this method includes the temporal evolution, it automatically also matches the shape of the hysteresis loop to the experimental data in the best possible way. The numerical effort for this multi-parameter least squares fit, which we intend to implement soon, is expected to be high, as it includes a large number of ASTRA runs.

Within the scope of this article, however, we restrict ourselves to the above ASTRA results obtained by manual parameter adjustment, which clearly illustrate that transient analysis has the potential of giving a deeper insight into the transport phenomena which are at play when the density profile shows a response to the application of central heating.

\section{SUMMARY AND OUTLOOK}

In this article, the transient behavior of density and temperature has been investigated in plasma discharges in which the application of central heat sources affects the shape of the density profile. Electron cyclotron heating has been used for this purpose, as this heating system allows for very localized power deposition and can access also the plasma center. It has been found that the temporal evolution of density and temperature at a given radial location follows a hysteresis curve, which occurs due to the fact that the temperature initially responds faster to changes in the applied heating power than the density. This observation holds for both, cases where central heating flattens the density profile (density pump-out), as well as for cases where central heating peaks the density profile. Special care with respect to the experimental conditions has been taken in order to avoid biasing factors which might affect the density profile as well.

In existing studies on density response to local heating, the focus of analysis is mostly only on the stationary density and temperature profiles. Just in [19], results from transient analysis are presented for FTU. The method applied there is based on Fourier transformation of density and temperature time traces and the comparison of their phase. In cases where central ECRH increases the core density, a phase delay of density relatively to temperature is reported. This is consistent with our observation. In case of sinusoidal time traces, a phase delay would give rise to the occurrence of a Lissajous ellipse in the density-temperature diagram, which is at least qualitatively pointing into the direction of the hysteresis loops we observe. For the density pump-out case, however, no phase delay between temperature and density is reported in [19]. At least within the data set provided by our series of experiments, no such case was found. We always observe a delayed response of density.

By performing ASTRA simulations, in which the transport parameters have been adjusted by hand in an iterative way, we have shown that the occurrence of hysteresis loops in the $n_{e}-T_{e}$ diagram can be reproduced on the basis of today's models for heat and particle transport. Already in this first approach, reasonable agreement between simulated and measured profiles was achieved. A numerical method of parameter adaption, whose implementation is within our future plans, is expected to yield more precise results. In the following experimental campaign on ASDEX Upgrade, we intend to continue our experiments making use of the increased heating power of the ECRH system, which has been upgraded by the installation of additional gyrotrons. These enhanced heating capabilities are of limited use in L-mode discharges, where the L-H threshold sets an upper limit to the amount of heating power that can be applied, but 
clearly extends the operational space for H-mode experiments. It is also foreseen to study the effect of non-central heating on the density profile by moving the ECRH deposition region radially outward.
[1] R. Gilgenbach, M. Read, K. Hackett, R. Lucey, B. Hui, V. Granatstein, K. Chu, A. England, C. Loring, O. Eldridge, H. Howe, A. Kulchar, E. Lazarus, M. Murakami and J. Wilgen, Physical Review Letters 44 647650 (1980).

[2] A. Riviere, Plasma Physics and Controlled Fusion 28 1263 (1986).

[3] C. Angioni, A.G. Peeters, X. Garbet, A. Manini, F. Ryter, and ASDEX Upgrade Team, Nuclear Fusion 44 827845 (2004).

[4] C. Angioni, A. Peeters, F. Ryter, F. Jenko, G. Conway, T. Dannert, H. Fahrbach, M. Reich, W. Suttrop and ASDEX Upgrade Team, Physics of Plasmas 12040701 (2005).

[5] E. Fable, C. Angioni, O. Sauter, Plasma Physics and Controlled Fusion 52015007 (2010).

[6] A. Mlynek, M. Reich, L. Giannone, W. Treutterer, K. Behler, H. Blank, A. Buhler, R. Cole, H. Eixenberger, R. Fischer, A. Lohs, K. Lüddecke, R. Merkel, G. Neu, F. Ryter, D. Zasche and the ASDEX Upgrade Team, Nuclear Fusion 51043002 (2011).

[7] R. Fischer, C. Fuchs, B. Kurzan, W. Suttrop, E. Wolfrum, and ASDEX Upgrade Team, Fusion Science and Technology 58 675-684 (2010).

[8] C. Angioni, R. McDermott, E. Fable, R. Fischer, T. Pütterich, F. Ryter, G. Tardini and the ASDEX Upgrade Team, Nuclear Fusion 51023006 (2011).

[9] G. Hoang, C. Bourdelle, X. Garbet, J. Artaud, V. Basiuk, J. Bucalossi, F. Clairet, C. Fenzi-Bonizec, C. Gil, J. Segui, J. Travere, E. Tsitrone and L. Vermare, Physical Review Letters 93135003 (2004).

[10] I. Furno, C. Angioni, F. Porcelli, H. Weisen, R. Behn, T. Goodman, M. Henderson, Z. Pietrzyk, A. Pochelon,
H. Reimerdes and E. Rossi, Nuclear Fusion 41 403-420 (2001).

[11] M. Willensdorfer, E. Wolfrum, R. Fischer, J. Schweinzer, M. Sertoli, B. Sieglin, G. Veres, F. Aumayr and the ASDEX Upgrade Team, Review of Scientific Instruments 83023501 (2012).

[12] G. Pereverzev and P. Yushmanov, 'ASTRA, Automated System for Transport Analysis in a Tokamak', IPP report $5 / 98$ (2002).

[13] C. Angioni, E. Fable, M. Greenwald, M. Maslov, A. Peeters, H. Takenaga, H. Weisen, Plasma Physics and Controlled Fusion 51124017 (2009).

[14] F. Imbeaux, F. Ryter and X. Garbet, Plasma Physics and Controlled Fusion 43 1503-1524 (2001).

[15] F. Ryter, G. Tardini, F. De Luca, H. Fahrbach, F. Imbeaux, A. Jacchia, K. Kirov, F. Leuterer, P. Mantica, A. Peeters, G. Pereverzev, W. Suttrop and the ASDEX Upgrade Team, Nuclear Fusion 43 1396-1404 (2003).

[16] H. Nordman, J. Weiland and A. Jarmen, Nuclear Fusion 30 983-996 (1990).

[17] A. Ware, Physical Review Letters 25 15-17 (1970).

[18] F. Ryter, R. Dux, P. Mantica and T. Tala, Plasma Physics and Controlled Fusion 52124043 (2010).

[19] A. Jacchia, S. Cirant, F. De Luca, P. Buratti, O. Tudisco, C. Mazotta, G. Calabro, G. Ramogida, C. Cianfarani, D. Marocco, G. Grossetti, G. Granucci, O. D'Arcangelo, $W$. Bin and FTU and ECRH team, $37^{\text {th }}$ EPS Conference on Controlled Fusion and Plasma Physics, Europhysics Conference Abstracts Vol.34A P1.1045 (2010), http://ocs.ciemat.es/EPS2010PAP/pdf/P1.1045.pdf. 